\title{
Efecto cicatrizante del aceite de Copaifera officinalis (copaiba), en pacientes con úlcera péptica
} Cicatrizing effect of Copaifera officinalis (copaiba) oil in patients with peptic ulcer

\author{
Jorge Arroyo-Acevedo ${ }^{1,2}$, Mariano Quino-Florentini ${ }^{3}$, Jaime Martínez-Heredia ${ }^{4}$, \\ Yuan Almora-Pinedo ${ }^{5}$, Alex Alba-González ${ }^{6}$, Martín Condorhuamán-Figueroa ${ }^{7}$ \\ 'Instituto de Investigaciones Clínicas, Facultad de Medicina, Universidad Nacional Mayor de San Marcos. Lima, Perú. \\ ${ }^{2}$ Laboratorio de Farmacología, Facultad de Medicina, Universidad Nacional Mayor de San Marcos, Lima, Perú. \\ ${ }^{3}$ Servicio de Gastroenterología, Hospital Nacional Dos de Mayo. Lima, Perú \\ ${ }^{4}$ Médico Asistente, Seguro Social de Salud (EsSalud), Lima, Perú. \\ ${ }^{5}$ Departamento de Farmacia, Hospital Nacional Hipólito Unanue, Lima, Perú. \\ ${ }^{6}$ Instituto de Ciencias Farmacéuticas y Recursos Naturales, Facultad de Farmacia y Bioquímica, Universidad Nacional Mayor de San \\ Marcos, Lima, Perú \\ Médico residente, Hospital Docente Materno Infantil San Bartolomé, Lima, Perú.
}

\begin{abstract}
Resumen
Objetivos: Determinar la eficacia cicatrizante del aceite de copaiba obtenido de la corteza de Copaifera officinalis, comparado con omeprazol $20 \mathrm{mg}$, en pacientes con diagnóstico definitivo de úlcera péptica. Diseño: Estudio experimental, clínico comparativo, de fase II, aleatorio, doble ciego, grupo paralelo. Institución: Instituto de Investigaciones Clínicas, Facultad de Medicina, Universidad Nacional Mayor de San Marcos, Lima, Perú. Participantes: Pacientes con diagnóstico definitivo de úlcera péptica. Intervenciones: El diagnóstico fue tanto por exploración física como complementaria, siendo la endoscopia la técnica de elección, con evaluación pre y postratamiento con aceite de copaiba, formulada en cápsulas de $80 \mathrm{mg}$ y $120 \mathrm{mg}$. El ensayo clínico incluyó 60 pacientes que voluntariamente ingresaron al programa de estudio, previa firma del consentimiento informado aprobado por el Comité institucional de Ética en Investigación. Los pacientes fueron distribuidos aleatoriamente en tres grupos, de 20 casos cada uno, según orden de llegada; los dos primeros grupos recibieron cápsulas de aceite de copaiba, en dosis de 80 y $120 \mathrm{mg}$, respectivamente; y un tercer grupo recibió omeprazol $20 \mathrm{mg}$. Los tratamientos fueron administrados en ayunas, una vez por la mañana, 30 minutos antes de la ingesta del primer alimento. Los datos fueron evaluados mediante técnicas multivariadas, considerando estadísticamente significativo $p<0,05$. Se tuvo en cuenta el consentimiento informado aprobado por el Comité de Bioética en Investigación del Centro Asistencial. Principales medidas de resultados: Porcentaje de cicatrización. Resultados: Se logró $65 \%$ y $75 \%$ de cicatrización de la úlcera péptica con aceite de copaiba, respectivamente, contra $100 \%$ en el grupo de omeprazol, sin efectos adversos significativos; dos presentaron náuseas y tres epigastralgia. Conclusiones: Los pacientes con úlcera péptica y con tratamiento de las cápsulas conteniendo aceite de copaiba mostraron cicatrización de la úlcera de 65 a $75 \%$ y sin efectos adversos significativos.
\end{abstract}

Palabras clave: Úlcera péptica, aceite de copaiba

\section{Abstract}

Objectives: To determine the cicatrizing effect of Copaifera officinalis' stem bark copaiba oil compared with omeprazole 20 mg in patients with diagnosis of peptic ulcer. Design: Experimental, comparative, phase II, randomized, double-blind, parallel-group clinical trial. Setting: Clinical Investigation Institute, Faculty of Medicine, Universidad Nacional Mayor de San Marcos, Lima, Peru. Participants: Patients with diagnosis of peptic ulcer. Interventions: Clinical diagnosis of peptic ulcer was done by endoscopy as well as pre and post treatment evaluation following administration of copaiba oil formulated capsules ( $80 \mathrm{mg}$ and $120 \mathrm{mg}$ ). Sixty patients enrolled voluntarily to the study and signed informed consent as approved by the Institutional Review Board. Patients were randomly distributed in three groups of 20 cases each according to arrival order; the first and second group received respectively copaiba oil $80 \mathrm{mg}$ and $120 \mathrm{mg}$ capsules, and the third group omeprazole $20 \mathrm{mg}$, fasting, once daily in the morning, half hour before breakfast. Data was evaluated through multivariate techniques, considering $p<0.05$ as statistically significant. Main outcome measures: Percentage of patients healing their ulcer. Results: Peptic ulcer cicatrized in $65 \%$ and $75 \%$ respectively versus $100 \%$ in the omeprazole group, without significant adverse effects. Two patients presented nausea and three epigastric pain. Conclusions: Patients with peptic ulcer treated with copaiba oil capsules showed ulcer scarring in $65 \%$ to $75 \%$ and without significant adverse effects.

Key words: Peptic ulcer, copaiba oil.

An Fac med. 2011;72(2):113-7 


\section{INTRODUCCIÓN}

La úlcera péptica, enfermedad crónica y recurrente de las enfermedades gastrointestinales, incide en $10 \%$ de la población ${ }^{(1)}$ y va en aumento, de acuerdo a como avanza la civilización ${ }^{(2)}$. La hemorragia digestiva alta es muy frecuente $(50 \%)$; la perforación y obstrucción ocurre en menos de $5 \%$ de pacientes con enfermedad ulcerosa ${ }^{(3)}$. La úlcera gástrica sería la nueva plaga del siglo XXI, por su estrecha relación con el cáncer gástrico (3\%) ${ }^{(4)}$. Son factores el estrés, fumar, deficiencias nutricionales, frecuente ingesta de AINES ${ }^{(5)}$. Existe evidencia de que el Helicobacter pylori es un agente desencadenante de la úlcera péptica; se acrecienta el cuadro sin tratamiento médico ${ }^{(6)}$.

La búsqueda de sustancias para el tratamiento de la úlcera péptica es necesaria. Los fármacos disponibles son costosos y con efectos adversos ${ }^{(7)}$. La óleo resina de Copaifera langsdorffi (familiar de Copaifera officinalis) evitó lesiones gástricas inducidas por etanol, indometacina y estrés por inmersión en hielo ${ }^{(8)} ; \mathrm{y}$, el aceite de copaiba de Copaifera officinalis, en animales de experimentación, redujo la secreción gástrica en ratas con ligadura pilórica, y cicatrizó lesiones gástricas inducidas por indometacina, corroborado por estudio histopatológico ${ }^{(9)}$.

Las especies de Copaifera son conocidas popularmente como 'copaiba', 'copa'iva' o 'pau-de-'oleo'. Desde el siglo 16 , el aceite de copaiba ha sido usado por los indios del norte y noreste del Brasil en el tratamiento de varias enfermedades. El amplio uso tradicional ha conllevado a la intensa comercialización de copaiba como aceite o en cápsulas ${ }^{(10)}$. Los efectos atribuidos al aceite de copaiba en medicina folclórica son antiiflamatorio, antitumor, antiséptico urinario, enfermedades de la piel, úlceras; también, como cicatrizante de heridas. Se ha estudiado las propiedades del aceite de copaiba, como antiiflamatoria ${ }^{(11)}$, antitumoral contra Walker sarcoma ${ }^{(12)}$ y línea celu- lar de melanoma ${ }^{(13)}$, antiulcerogénico, antioxidante y antiloperoxidativo (8), cercaricida, antihelmíntico ${ }^{(14)}$ y antimicrobiano ${ }^{(15)}$. También, como repelente de insectos ${ }^{(16)}$ y en la industria cosmética en la fabricación de lociones capilares y shampú ${ }^{(17)}$.

La hipótesis del presente trabajo ha sido que la administración oral de cápsulas conteniendo aceite de copaiba -producto natural- cicatriza las úlceras en los pacientes con úlcera péptica y con mínimos efectos adversos. Han sido los objetivos determinar el efecto cicatrizante al administrar cápsulas conteniendo el aceite de copaiba por vía oral durante 30 días a pacientes con úlcera péptica, evaluar los posibles efectos adversos y comparar el efecto de las cápsulas conteniendo aceite de copaiba con cápsulas de omeprazol 20 $\mathrm{mg}$, al ser administradas durante 28 días de tratamiento en pacientes con úlcera péptica.

\section{MÉTODOS}

Se formuló cápsulas con 80 y 120 mg de aceite de copaiba, conteniendo excipientes inertes, como aerosil y veegum, en cantidades suficientes y permitidas por la farmacopea de los Estados Unidos. En todos los casos se tuvo la precaución que la administración de las cápsulas de aceite de copaiba fuera por la mañana, 30 minutos antes de la ingesta de los alimentos.

Se enroló un total de 60 pacientes con el diagnóstico de úlcera péptica, que voluntariamente ingresaron al estudio, previa autorización y firma del consentimiento informado aprobado por el Comité Institucional de Ética en Investigación del Hospital Nacional Dos de Mayo, Lima, Perú. Los pacientes fueron distribuidos según orden de llegada, de acuerdo al diseño experimental mostrado en la tabla 1.

La evaluación se realizó mediante estudio clínico (signos y síntomas propios de la enfermedad), endoscópico (tamaño, número y ubicación de la úlcera) y biópsico (para detectar presencia de Helicobacter pylori y de proceso neoformativo). Los pacientes con tratamiento según diseño fueron sometidos a evaluación a los 15 días y nuevamente a los 30 días postratamiento, para observar cicatrización de la úlcera duodenal y gástrica. Para el estudio de seguridad, se hizo seguimiento observacional de signos y síntomas adversos que pudieran presentarse con la terapia farmacológica, como intolerancia gástrica (náuseas, vómitos, dolor abdominal); también, se evaluó por estudios bioquímicos (glicemia, urea, creatinina, perfil hepático, proteínas totales, albúmina, bilirrubina, transaminasas, fosfatasa alcalina) y hematológicos (hemograma completo, velocidad de sedimentación).

Se incluyó los pacientes con diagnóstico definitivo clínico y endoscópico de úlcera péptica, de 18 a 80 años de edad, de ambos sexos. Se excluyó los pacientes complicados (procesos neoformativos, alteraciones hepáticas severas, alteraciones renales y alteraciones cardiovasculares), ingesta previa de fármacos bloqueadores $\mathrm{H} 2$, inhibidores de bomba, antiácidos, citoprotectores 48 horas antes de haber presentado el diagnóstico de úlcera péptica; además, los pacientes con contraindicación para endoscopia (insuficiencia coronaria aguda, obstrucciones definidas del esófago superior), con antecedentes

Tabla 1. Diseño experimental de los pacientes con úlcera péptica (UP).

\begin{tabular}{|c|c|c|}
\hline$N^{\circ}$ & Tratamiento & N \\
\hline 1 & Pacientes con UP + aceite de copaiba $80 \mathrm{mg} /$ día & 20 \\
\hline 2 & Pacientes con UP + aceite de copaiba $120 \mathrm{mg} /$ día & 20 \\
\hline 3 & Pacientes con UP + omeprazol $20 \mathrm{mg} /$ día & 20 \\
\hline
\end{tabular}


de alergia a cualquier bloqueador $\mathrm{H} 2 \mathrm{o}$ inhibidor de la bomba de protones, embarazadas y lactantes, así como, aquellos pacientes en los que mediante el examen físico y de laboratorio se había descartado otras alteraciones que no correspondían con úlcera péptica.
Para el análisis estadístico, se incluyó tabulaciones descriptivas de las características clínicas de los pacientes, estimación de la media y desviación estándar de valores -antes y después del tratamiento-; se determinó la variación de la cicatrización de úlceras en los pa-

Tabla 2. Características y clínica de los pacientes con úlcera péptica, según tratamiento. $n=20$. Entre paréntesis, porcentajes.

\begin{tabular}{|c|c|c|c|}
\hline & $\begin{array}{l}\text { Inhibidores bomba } \\
\text { protones }\end{array}$ & Aceite copaiba $80 \mathrm{mg}$ & Aceite copaiba $120 \mathrm{mg}$ \\
\hline \multicolumn{4}{|l|}{ Edad (años) } \\
\hline$<20$ & 1 & 2 & 0 \\
\hline 21 a 30 & 7 & 6 & 9 \\
\hline 31 a 40 & 5 & 5 & 5 \\
\hline 41 a 60 & 5 & 6 & 4 \\
\hline$>60$ & 2 & 1 & 2 \\
\hline$p$ & 0,600 & 0,450 & 0,000 \\
\hline \multicolumn{4}{|l|}{ Sexo } \\
\hline Hombres & $15(75)$ & $15(75)$ & $17(85)$ \\
\hline Mujeres & $5(25)$ & $5(25)$ & $3(15)$ \\
\hline$p$ & 0,000 & 0,002 & 0,000 \\
\hline \multicolumn{4}{|l|}{ Síntomas } \\
\hline Ninguno & 0 & 0 & 0 \\
\hline Dispepsia & 6 & 6 & 8 \\
\hline Epigastralgia & 12 & 8 & 10 \\
\hline Náusea y llenura & 2 & 3 & 0 \\
\hline Síndrome ulceroso & 0 & 3 & 2 \\
\hline$p$ & 0,600 & 0,360 & 0,600 \\
\hline \multicolumn{4}{|l|}{ Signos } \\
\hline Ninguno & 10 & 6 & 7 \\
\hline Palidez & 4 & 6 & 5 \\
\hline $\begin{array}{l}\text { Hemorragia } \\
\text { (melena - } \\
\text { hematemesis) }\end{array}$ & 6 & 5 & 5 \\
\hline Dolor epigástrico & 5 & 6 & 6 \\
\hline Vómitos, náuseas & 4 & 3 & 2 \\
\hline Shock & 3 & 0 & 0 \\
\hline Total & $32^{*}$ & $25^{*}$ & $25^{*}$ \\
\hline$p$ & 0,670 & 0,487 & 0,670 \\
\hline \multicolumn{4}{|l|}{$\begin{array}{l}\text { Tiempo de enfermedad } \\
\text { (semanas) }\end{array}$} \\
\hline$<2$ & $12(60)$ & $6(30)$ & $11(55)$ \\
\hline $2-4$ & $4(20)$ & $6(30)$ & $4(20)$ \\
\hline $4-8$ & $2(10)$ & $3(15)$ & $3(15)$ \\
\hline$>8$ & $2(10)$ & $5(25)$ & $2(10)$ \\
\hline$p$ & 0,500 & 0,007 & 0,000 \\
\hline \multicolumn{4}{|l|}{$\begin{array}{l}\text { Antecedentes de } \\
\text { enfermedad }\end{array}$} \\
\hline Ninguno & 11 & 11 & 14 \\
\hline Familiares & 2 & 3 & 0 \\
\hline Alcohol & 3 & 2 & 1 \\
\hline $\begin{array}{l}\text { Patologías } \\
\text { asociadas }\end{array}$ & 2 & 2 & 3 \\
\hline Fármacos (AINES) & 2 & 2 & 2 \\
\hline$p$ & 0,160 & 0,230 & 0,000 \\
\hline
\end{tabular}

* Algunos de los pacientes presentaron más de un signo.

cientes que recibieron aceite de copaiba u omeprazol $20 \mathrm{mg}$ al día. Se realizó comparaciones estadísticas de las características clínicas de los pacientes entre ambos grupos de tratamiento, mediante las pruebas chi-cuadrado o exacta de Fisher, y la variación (diferencia entre pre y post) de la eficacia cicatrizante entre ambos grupos mediante la estadística $\mathrm{t}$-student o prueba $\mathrm{F}$ obtenida a través del análisis de varianza para medidas repetidas. Todas las evaluaciones fueron realizadas a un nivel de significancia de 5\%. Los datos fueron procesados mediante el programa SPSS versión 13, año 2006. La recolección de la información fue efectuada antes del inicio de la terapia y después de ser sometidos al tratamiento con aceite de copaiba procedente de Copaifera officinalis, en cápsulas de 80 y 120 mg, en protocolos y formatos de estudio elaborados para el presente proyecto.

El consentimiento informado fue obtenido de cada paciente que voluntariamente ingresó al programa; el proyecto fue sometido a consideración del Comité de Ética del centro asistencial y del Comité de Expertos.

\section{RESULTADOS}

Los pacientes elegibles tuvieron entre 18 y 80 años, con promedio de 50 años de edad, siendo hombres entre 75 y $85 \%$ y mujeres entre 15 y $25 \%$. En la tabla 2 observamos las características y signos y síntomas de los pacientes. Durante el estudio no se permitió otro tratamiento.

En la tabla 3, se muestra los resultados de la endoscopia, de acuerdo a las escalas de Sakita y Forrest, y en la tabla 4, los resultados de la endoscopia de control y de la biopsia.

Con relación a la tolerabilidad, dos de los pacientes que recibieron el producto natural presentaron náuseas y tres epigastralgia. El resto de los pacientes no tuvo evento adverso durante el estudio clínico, que hubiera requerido suspensión del estudio. 
Tabla 3. Endoscopia inicial según escala de Sakita y de Forrest.

\begin{tabular}{|c|c|c|c|}
\hline & $\begin{array}{l}\text { Inhibidores bomba } \\
\text { protones }\end{array}$ & Aceite copaiba 80 mg & Aceite copaiba $120 \mathrm{mg}$ \\
\hline \multicolumn{4}{|c|}{ Según escala de Sakita } \\
\hline Sakita A & 15 & 11 & 13 \\
\hline Sakita H & 7 & 8 & 7 \\
\hline Sakita S & 2 & 3 & 1 \\
\hline Total & 24 & 22 & 21 \\
\hline$p$ & 0,000 & 0,005 & 0,000 \\
\hline \multicolumn{4}{|c|}{ Según escala de Forrest } \\
\hline Forrest I & 0 & 0 & 0 \\
\hline Forrest II & 7 & 6 & 5 \\
\hline Forrest III & 18 & 14 & 17 \\
\hline Total & 25 & 20 & 22 \\
\hline$p$ & 0,000 & 0,007 & 0,000 \\
\hline
\end{tabular}

Tabla 4. Endoscopia de control y resultados de la biopsia. Entre paréntesis, porcentajes.

\begin{tabular}{|c|c|c|c|}
\hline \multirow{2}{*}{$\begin{array}{c}\text { Cicatrización } \\
\text { Cicatrizaron }\end{array}$} & $\begin{array}{c}\text { Inhibidores bomba } \\
\text { protones }\end{array}$ & $\begin{array}{c}\text { Aceite copaiba } 80 \mathrm{mg} \\
\text { Aceite copaiba } 120 \mathrm{mg}\end{array}$ \\
\hline No cicatrizaron & $20(100)$ & $13(65)$ & $15(75)$ \\
\hline$p$ & 0 & $7(35)$ & $5(25)$ \\
\hline Biopsia & 0,000 & 0,011 & 0,000 \\
\hline Gastritis (+) & $\begin{array}{c}\text { Inhibidores bomba } \\
\text { protones }\end{array}$ & Aceite copaiba $80 \mathrm{mg}$ & Aceite copaiba $120 \mathrm{mg}$ \\
\hline Gastritis (-) & $20(100)$ & $20(100)$ & $20(100)$ \\
\hline Helicobacter pylori (+) & $20(100)$ & $8(40)$ & $10(50)$ \\
\hline Helicobacter pylori (-) & $20(100)$ & $20(100)$ & $8(100)$ \\
\hline$p$ & $0(100)$ & $6(30)$ & 0,500 \\
\hline
\end{tabular}

\section{DISCUSIÓN}

Atendiendo a las características físicoquímicas del extracto a tratar y en virtud al inconveniente de poder obtener un extracto seco del mismo, se eligió excipientes que pudieran favorecer su encapsulación, siendo ellos Veegum HV y el Aerosil 200, el primero de retención de sustancias orgánicas por su capacidad de intercambio iónico, además de su capacidad aglutinante; y el segundo por su gran poder absorbente. Ambos, son silicatos cuya absorción por vía intestinal es despreciable o nula ${ }^{(25,26)}$.

La ulceración gastrointestinal y la úlcera péptica son causas significativas de morbilidad en los Estados Unidos y muchos otros países. La medicaciones de procesos inflamatorios son también causa de la patogénesis de ulceraciones, como en los trastornos reumatológicos, donde es necesario el uso de AINEs, los que incrementan el riesgo de la enfermedad; por ello es necesario el uso de la terapia asociada con inhibidores de la bomba de protones y en algunos casos antibióticos, si se trata de una infección por $H$. pylori ${ }^{(27,28)}$.

En los ensayos fitoquímicos realizados al aceite de copaiba, se identificó la presencia de terpenos ${ }^{(9)}$, sin descartar la posibilidad que en su composición contenga mayor cantidad de triterpenos; dentro de estos, el $\beta$-cariofileno se encuentra en mayor cantidad, seguido de $\alpha$-humuleno, $\alpha$-copaeno, $\alpha$-bergamoteno, $\delta$-cadineno. Cabe resaltar que el $\beta$-cariofileno ha mostrado efecto citoprotector gástrico ${ }^{(29)}$, afectando la secreción de ácido clorhídrico y pepsina, además de efectos antiinflamatorios, sin ninguna manifestación de daño de la mucosa gástrica. Esto es por ser un potente inhibidor selectivo de la bomba de protones de las células parie- tales del estómago, por lo que reduce la secreción del ácido gástrico, por inhibición de la enzima $\mathrm{H}+/ \mathrm{K}+$ ATPasa, uniéndose a la bomba de protones localizada en la membrana de las células parietales gástricas. Además, bloquea el transporte activo de los iones hidrógeno e incrementa el $\mathrm{pH}$ gástrico ${ }^{(9)}$, lo que explica los hallazgos expresados en la tabla 4, donde se observa que el aceite de copaiba ha mostrado un efecto cicatrizante del proceso ulceroso, sin descartar el mejor efecto del omeprazol.

Es bien conocido que dentro de los componentes presentes en el aceite de copaiba se encuentra un diterpeno llamado ácido kaurénico ${ }^{(30)}$. Cabe mencionar que el grupo kaureno de los diterpenos (incluyendo el ácido kaurénico) parecen afectar la señal inflamatoria a través de la inhibición de factores nucleares, como el factor nuclear-kappaB (FN-kB), regulando la trascripción de varias citoquinas inflamatorias ${ }^{(31)}$.

En este sentido, debido a que las citoquinas y los factores de crecimiento regulados por $\mathrm{FN}-\mathrm{kB}$ son necesarios para la curación de heridas, no es deseable una regulación descendente completa de FN-kB, sino una disminución evidente en su regulación ascendente. Por lo tanto, aquellos extractos vegetales que disminuyen moderadamente la activación $\mathrm{FN}-\mathrm{kB}$ pueden contribuir a los procesos de curación y cicatrización de heridas ${ }^{(32)}$. Estos procesos moleculares que explican la cicatrización de heridas estarían justificando el efecto encontrado con aceite de copaiba en cápsulas, utilizado en los pacientes con úlcera péptica.

Por otro lado, el ácido kaurénico presente en el aceite de copaiba ${ }^{(30)}$, según estudios, es un compuesto diterpenoide que posee actividad antibacteriana frente a Staphylococcus aureus, Bacillus cereus y Bacillus subtilis, con un MIC de $10 \mu \mathrm{g} / \mathrm{mL}^{(33)}$. También, se ha mostrado efecto antimicrobiano significativo (MIC $<10 \mu \mathrm{g} / \mathrm{mL}$ ) contra bacterias gram-positivas, comparable con cefotaxima usado como control ${ }^{(34)}$. Se debe considerar que los análisis fitoquí- 
micos de la fracción volátil de Centella asiática, una planta medicinal que se desarrolla en África del Sur, ha demostrado que contiene gran cantidad de $\alpha$-humuleno, $\beta$-cariofileno y bicyclogermacreno, y que el estudio microbiológico de estos ingredientes indica un gran efecto contra microorganismos gram positivos y gram negativos ${ }^{(35)}$.

El efecto antibacteriano encontrado para el ácido kaurénico $\beta$-cariofileno, componente del aceite de copaiba, podría contribuir en la reducción del Helicobacter pylori observado en el presente estudio (tabla 4), lo que estaría acorde a la literatura científica, donde se destaca los efectos antiinflamatorio y antibiótico del aceite de copaiba ${ }^{(36,37)}$. Se recomienda proseguir estudios con mayor número de pacientes, considerar pacientes con úlcera gástrica, úlcera duodenal, estudios in vitro para evaluar la eficacia frente al Helicobacter pylori (HP) o identificar la presencia de HP con pruebas específicas de diagnóstico por métodos no invasivos, como la prueba del aliento de la urea.

Se llega a la conclusión de que, los pacientes con úlcera péptica y con tratamiento de las cápsulas conteniendo aceite de copaiba han mostrado cicatrización de la úlcera de 65 a $75 \%$ y sin efectos adversos significativos.

\section{REFERENCIAS BIBLIOGRÁFICAS}

1. Rodríguez-Hernández $\mathrm{H}$, Jacobo-Karam JS, Guerrero-Romero F. Factores de riesgo para la recurrencia de úlcera péptica. Gac Méd Méx. 2001;137(4):303-3.

2. Guyton AC, Hall JE, Fisiologia de trastornos digestivos. Tratado de Fisiologia Médica. Unidad XII. Capítulo 66. Elsevier. 2006:819-25.

3. Chan CY, Yau KK, Siu WT, Wong KH, Luk YW, Tai TY, Li KW. Endoscopic hemostasis by using the TriClip for peptic ulcer hemorrhage: a pilot study. Gastrointest Endosc. 2008;67(1):35-9.

4. O'Malley P. Gastric ulcers and GERD: the new "plagues" of the 21st century update for the clinical nurse specialist. Clinical Nurse Specialist. 2003:17:286-9.

5. Belaiche J, Burette A, De Vos M, Louis E, Huybrechts M, Deltenre M. Observational survey of NSAIDrelated upper gastro-intestinal. Acta Gastroenterol Belg. 2002;65(2):65-73.

6. Chan FK, To KF, Wu JC, Yung MY, Leung WK, Kwok $T$, Hui Y. Eradication of Helicobacter pylori and risk of peptic ulcers in patients starting long-term treatment with nonsteroidal anti-inflammatory drugs a randomised trial. Lancet. 2002:359:9-13.

7. Rates SMK. Plants as source of drugs. Toxicol. 2001;39:603-13.

8. Paiva LAF, Gurgel LA, Campos AR, Silveira ER, Rao VSN. Attenuation of ischemia/reperfusion-induced intestinal injury by oleo-resin from Copaifera langsdorffii in rats. Life Sciences. 2004;75:1979-87.

9. Arroyo J, Almora Y, Quino M, Martínez J, Condorhuamán M, Flores M, Bonilla P. Efecto citoprotector y antisecretor del aceite de Copaifera officinalis en lesiones gástricas inducidas en ratas. An Fac med. 2009;70(2):89-96.

10. Veiga VF, Zunino L, Calixto JB, Patitucci ML, Pinto AC. Phytochemical and antioedematogenic studies of commercial Copaiba oils available in Brazil. Phytotherapy Research. 2001;15:476-80.

11. Basile AC, Sertie JA, Freitas PCD, Zanini AC. Anti-inflammatory activity of oleoresin from Brazilian Copaiba. J Ethnopharmacol. 1998;22:101-9.

12. Ohsaki A, Yan LT, Shigeru I, Edatsugi H, Iwata D, Komoda Y. The isolation and in vivo potent antitumor activity of clerodane diterpenoid from de oleoresin of the Brazilian medicinal plant, Copaiba. Bioorganic and Medicinal Chemistry Letters. 1994;4:2889-92.

13. Lima SRM, Veiga VF, Christo HB, Pinto AC, Fernandes PD. In vivo and in vitro studies on the anticancer activity of Copaifera multijuga Hayne and its fractions. Phytotherapy Research. 2003;17:1048-53.

14. Gilbert B, Mors WB, Baker PM, Tomassini TCB, Goulart EG, Holanda JC, et al. A atividade antihelmıntica de oleos essenciais e de seus componentes quımicos. Anais da Academia Brasileira de Ciencias. 1972;44:423-8.

15. Kang R, Helms R, Stout MJ, Jaber H, Chen Z, Nakatsu T. Antimicrobial activity of the volatile constituents of Penilla frutescens and its synergistic effects with polygodial. J Agric Food Chem. 1992;40:2328-30.

16. Lacey LA, Schreck CE, McGovern TP. Native and experimental repellent against black flies in the Amazon basis of Brazil. Mos News. 1981;41:376-9.

17. Del Nunzio MJ. Copaiba oils and its uses in cosmetics. Aerosol Cosmetology. 1985;7:7.

18. Valle DL. Peptic ulcer diseases and related disorders. En: Braunwald E, Fauci AS, Kasper DL, Hauser SL, Longo DL, Jameson JL, editors. Harrison's principles of internal medicine. 16th ed. New York: McGraw-Hill; 2005:1746-62.

19. Ford AC, Delaney BC, Forman D, Moayyedi P. Tratamiento erradicación de úlcera péptica en pacientes pruebas positivas a Helicobacter pylori. Biblioteca Cochrane Plus, número 3, 2008. http:// www.update-software.com. (15/11/08).

20. Lewis JD, Bilker WB, Brensinger C, Farrar JT, Strom BL. Hospitalization and mortality rates from peptic ulcer disease and Gl bleeding in the 1990s: relationship to sales of nonsteroidal anti-inflammatory drugs and acid suppression medications. Am J Gastroenterol. 2002;97:2540-9.

21. Ohmann C, Imhof M, Ruppert C, Janzik U, Vogt C Frieling $T$, et al. Time-trends in the epidemiology of peptic ulcer bleeding. Scand J Gastroenterol. 2005;40:914-20.

22. Viviane A, Alan BN. Estimates of costs of hospital stay for variceal and nonvariceal upper gastrointestinal bleeding in the United States. Value Health. 2008;11:1-3

23. Garcia CV, Nudelman NS, Steppe M, Schapoval EES. Structural elucidation of rabeprazole sodium photodegradation products. J Pharm Biomed Analysis. 2008;46:88-93.

24. Falca HS, Mariath IR, Diniz MFFM, Batista LM, Barbosa-Filho JM. Plants of the American continent with antiulcer activity. Phytomedicine. 2008;15:132-46.
25. Fauli T. Tratado de Farmacia Galénica. Primera edición.. Madrid: Ed. Luzan 5 S.A.1993:143-62.

26. USP. The United States Pharmacopeia USP 28. 2005.

27. Lewis SC, Langman MJ, Laporte JR, Matthews JN, Rawlins MD, Wiholm BE. Dose-response relationships between individual nonaspirin nonsteroidal anti-inflammatory drugs (NANSAIDs) and serious upper gastrointestinal bleeding: a meta-analysis based on individual patient data. Br J Clin Pharmacol. 2002;54(3):320-6.

28. Mellemkjaer L, Blot WJ, Sorensen HT, Thomassen L, McLaughlin JK, Nielsen GL, Olsen JH. Upper gastrointestinal bleeding among users of NSAIDs: a population-based cohort study in Denmark. $\mathrm{Br} \mathrm{J}$ Clin Pharmacol. 2002;53(2):173-81.

29. Tambe Y, Tsujiuchi H, Honda G, Ikeshiro Y, Tanaka S. Gastric cytoprotection of the non-steroidal anti-inflammatory sesquiterpene, $\beta$-caryophyllene. Planta medica. 1996;62(5):469-70.

30. Paiva LAF, Gurgel LA, Silva RM, Tome AR, Gramosa NV, Silveira ER, Santos FA, Rao VSN. Antiinflammatory effect of kaurenoic acid, a diterpene from Copaifera langsdorfii on acetic acid-induced colitis in rats. Vascular Pharmacol. 2002;39:303-7.

31. Haas AF, Wong JW, Iwahashi CK, Halliwell B, Cross $\mathrm{CE}$, Davis PA. Redox regulation of wound healing? NF- $\kappa B$ activation in cultured human keratinocytes upon wounding and the effect of low energy $\mathrm{HeNe}$ irradiation. Free Radical Biol Med. 1998;25(9):9981005

32. Schmidt C, Fronza M, Goettert M, Geller F, Luik S, Flores EMM, Bittencourt CF. Biological studies on Brazilian plants used in wound healing. J Ethnopharmacol. 2009;122(3):523-32.

33. Van Vuuren SF. Antimicrobial activity of South African medicinal plants. J Ethnopharmacol. 2008;119:462-72.

34. Tincusi BM, Jimenez IA, Bazzocchi IL, Moujir LM, Mamani ZA, Barroso JP, et al. Antimicrobial terpenoids from the oleoresin of the Peruvian medicinal plant Copaifera paupera. Planta Medica. 2002;68:808-12.

35. Zheng CJ, Qin LP. Chemical components of Centella asiatica and their bioactivities. Zhong Xi Yi Jie He Xue Bao. 2007;5(3):348-51.

36. Coelho-Ferreira M. Medicinal knowledge and plant utilization in an Amazonian coastal community of Marudá, Pará State (Brazil). J Ethnopharmacol. 2009;126:159-75.

37. Ferreira de Lima MR, Luna JS, Feitosa dos Santos A, Caño de Andrade MC, Goulart Sant'Ana AE, Genet JP, Marquez B. Anti-bacterial activity of some Brazilian medicinal plants. J Ethnopharmacol. 2006;105:137-47.

\section{Financiamiento:}

Consejo Superior de Investigaciones UNMSM

Conflictos de intereses:

Ninguno

Trabajo presentado a las IX Jornadas Científicas Sanfernandinas, XII Jornadas de Investigación en Salud, XIX Jornadas Sanfernandinas Estudiantiles, setiembre 2010

\section{Correspondencia:}

Jorge Arroyo Acevedo

Correo electrónico: jorgearroyo54@yahoo.es 